\title{
Classificação de alunos desanimados em um AVEA: uma proposta a partir da mineração de dados educacionais
}

\author{
Fabrícia Damando Santos ${ }^{1,2}$, Magda Bercht ${ }^{1}$, Leandro Krug Wives ${ }^{1}$ \\ ${ }^{1}$ PGIE - Universidade Federal do Rio Grande do Sul (UFRGS) \\ ${ }^{2}$ Universidade Estadual do Rio Grande do Sul (UERGS) \\ fabriciadamando@gmail.com, berchteinf.ufrgs.br, wives@inf.ufrgs.br
}

\begin{abstract}
This paper reports a research that identifies the student discouraged in a virtual learning environment using data mining. An experiment was conducted to test the feasibility of adopting a predictive model in a regular discipline. The data classification was performed using the decision tree technique, and the results of the predictive model were correct in $91 \%$ of the data. Scherer's definitions were used for discouragement specification. New experiments will be conducted to validate the model and develop actions that assist the teacher to support these students.
\end{abstract}

Resumo. $O$ artigo relata uma pesquisa que identifica o aluno desanimado em um ambiente virtual de ensino e aprendizagem utilizando mineração de dados. Foi realizado um experimento para testar a viabilidade de adoção de um modelo preditivo em uma turma de uma disciplina regular. Foi feita a classificação dos dados utilizando a técnica de árvore de decisão, e os resultados do modelo preditivo mostraram acerto em cerca de $91 \%$ dos dados. Foram usadas definições de Scherer para a especificação do desanimo. Novos experimentos serão realizados a fim de validar o modelo e desenvolver ações que subsidiem o professor no apoio a esses alunos.

\section{Introdução}

Este trabalho apresenta a descrição de um experimento, parte de uma pesquisa, que pretende investigar e mostrar a importância em considerar a dimensão afetiva no contexto educacional, principalmente relacionado ao aluno desanimado. A pesquisa prevê um experimento com a aplicação de técnicas de Mineração de Dados Educacionais (MDE), com o intuito de apresentar um modelo de classificação de alunos propensos ao desânimo. Dessa forma, foi feito um experimento com alunos da disciplina Análise de Demonstrações Contábeis ofertada pelo Departamento de Ciências Contábeis e Atuárias da Universidade Federal do Rio Grande do Sul (UFRGS), a fim de apresentar um modelo de classificação de alunos propensos ao desânimo.

A escolha pelo estado afetivo de desânimo se deu pelo fato dele ser percebido como um estado onde há uma tendência de conduzir o aluno à desistência do curso (Longhi, 2011). Essa situação é mais presente em cursos a distância, devido à quebra da simultaneidade espaço-temporal e a dificuldade do professor em identificar os alunos desanimados, necessitando de subsídio para essa inferência. Nesse sentido, algumas iniciativas estão sendo realizadas no que diz respeito à análise de dados dos ambientes virtuais de aprendizagem (AVEA), coletando dados comportamentais e aplicando as 
técnicas de MDE, de modo a gerar subsídios para o apoio ao professor (Rigo et al., 2014; Majadas, Santos e Boticário 2013).

Os aspectos afetivos e comportamentais dos alunos podem ser obtidos através de suas interações no AVEA ao utilizar as ferramentas do ambiente e deixar registradas as ações no ambiente. Ações e dados comportamentais como o tempo de demora em fazer uma atividade, o número de erros que ocorre na execução de uma atividade, o número de solicitações de ajuda, a forma de se expressar em textos, podem apoiar na identificação dos estados afetivos do aluno (Bercht, 2001).

Este trabalho está estruturado da seguinte forma: a seção 2 aborda as noções que fundamentam o trabalho, abordando tanto os aspectos afetivos ligados à educação, bem como a importância e técnicas da mineração de dados educacionais. A seção 3 apresenta trabalhos relacionados. A seção 4 descreve o procedimento metodológico. A seção 5 detalha a análise e resultados do experimento. Finaliza-se com a seção 6 ao apresentar a conclusão.

\section{Referencial Teórico}

\subsection{Computação Afetividade e sua abordagem na educação}

Há mais de uma década a Computação Afetiva (CA) foi apresentada por Picard (2000) devido à preocupação em fazer com que computadores pudessem reconhecer emoções, expressar emoções e responder adequadamente às emoções de quem interagisse com ele, devido ao fato das emoções serem essenciais no processo de tomada de decisão, na percepção, influenciando o aprendizado além da memória e processos cognitivos.

Uma das áreas de aplicação da CA é a Educação, visto que a CA pode auxiliar na solução dos grandes desafios da aprendizagem ao utilizar métodos e técnicas que permitam desenvolver programas que reconheçam emoções, possam subsidiar o professor, prover assistência personalizada ao aluno, dentre outras (Picard, 2000; Jaques e Nunes, 2012; Baker, Isotani, Carvalho, 2011; Iepsen, 2013, Rigo et al, 2014). Dessa forma, abordar características psicológicas humanas para o ambiente virtual, basicamente relacionado aos afetos, proporciona diminuição do gap existente entre o ensino presencial e o virtual, principalmente no que diz respeito à $\mathrm{CA}$, conforme já mencionavam Jaques e Nunes (2012).

Diante disso, aspectos relacionados à afetividade como os estados de ânimo passam a ser pesquisados, pois são fenômenos afetivos que tem uma maior duração no tempo e podem ser observados mais comumente nas atividades de aprendizagem dos alunos e, principalmente, devido a sua importância nos processos de aprendizagem (Longhi, 2011; Scherer, 2005).

Os estados de ânimo foram fundamentados a partir de pesquisas de Scherer (2005) onde o autor desenvolveu um instrumento, que está na sua segunda versão, intitulado de Geneva Emotion Whell (GEW). A GEW 2.0 é representada em formato de roda a qual divide os estados de ânimo em quatro categorias (animado, desanimado, satisfeito e insatisfeito), abrangendo um total de 20 famílias afetivas. A GEW é um instrumento confiável usada como método de obtenção do auto-relato para inferência dos estados de ânimo. A GEW 2.0 foi adaptada para um formato em português e intitulada de Roda de Estados de ânimo (REA 2.0) para ser usada como auto-relato pelo 
aluno, frente ao seu sentimento relacionado à várias situações de aprendizagem (Damando e Bercht, 2014; Longhi 2011).

Através da REA 2.0 o aluno pode selecionar uma das 20 famílias afetivas e sua intensidade, que varia de acordo com o tamanho do círculo que acompanha a família afetiva. A REA 2.0 pode ser observada na figura 1.

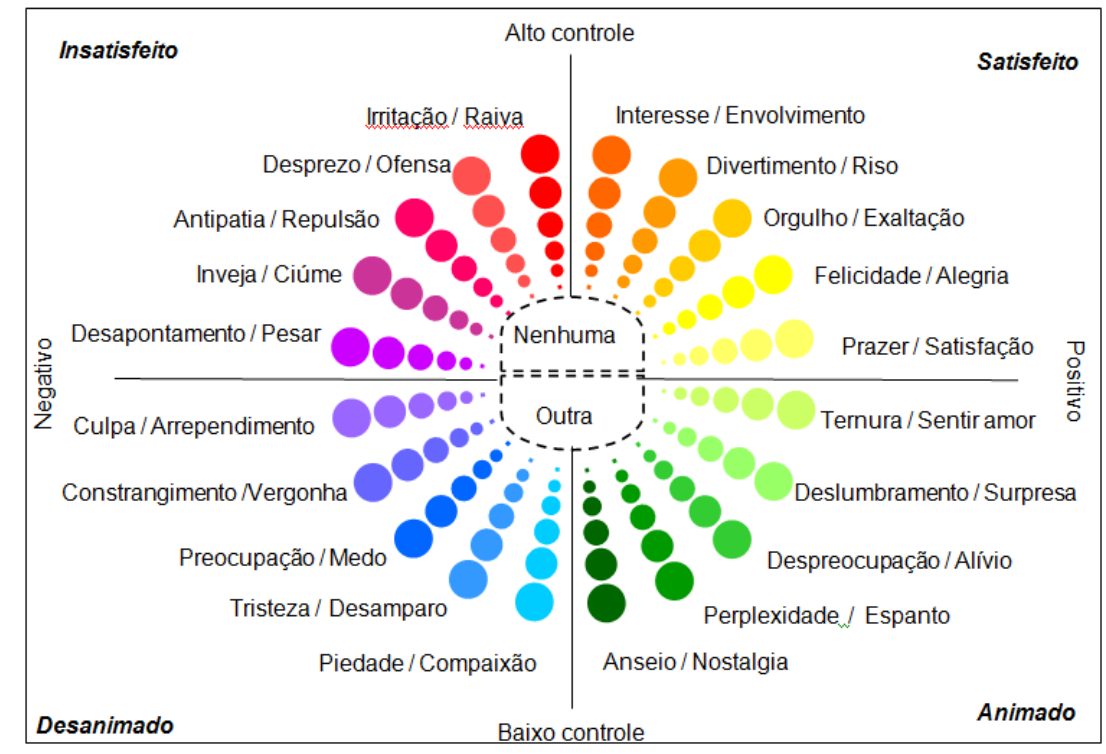

Figura 1: Roda de Estados de Ânimo 2.0 adaptada pelos autores a partir de Sacharin, Schlegel e Scherer (2013) e Longhi (2011)

Dentre os estados de ânimo classificados por Scherer (2005), o desânimo é percebido como um estado onde há uma tendência de conduzir o aluno à desistência ou submissão, porém, é um estado que possibilita reversão, fazendo com que o aluno possa retomar seu processo de aprendizagem (Longhi, 2011) justificando, assim, a sua abordagem no presente trabalho.

Para identificar um estado de ânimo, torna-se necessário observar variáveis comportamentais dos alunos dentro do ambiente. São consideradas como variáveis comportamentais as ações que são passíveis de serem identificadas e que são consideradas como importantes definidoras do comportamento do aluno, podendo ser usadas como indicadores para identificar o seu estado afetivo (Bercht, 2001). No presente trabalho, foram usadas as variáveis comportamentais vindas dos logs do AVEA Moodle, como por exemplo, quantidade de acessos aos fóruns, quantidade de postagens e visualizações.

Uma das dificuldades para a análise das variáveis comportamentais existentes nos AVEA se deve ao grande volume de dados gerados. Porém, aplicar técnicas de MDE sobre esses dados podem revelar informações importantes, como o caso de predizer e classificar aspectos relacionados à afetividade do aluno (Pardos et al., 2014; Iepsen, 2012; Inventado et al., 2013). 


\subsection{Mineração de dados educacionais}

Mineração de Dados Educacionais (MDE) é uma área de pesquisa cujo principal foco é desenvolver e/ou adaptar métodos e algoritmos de mineração para explorar conjunto de dados produzidos e coletados em ambientes educacionais (Baker, Isotani e Carvalho, 2011).

Dessa forma, a MDE busca compreender dados em contextos educacionais produzidos por alunos, professores e suas interações, na tentativa de responder perguntas específicas da educação. A MDE pode ser aplicada, por exemplo, para verificar a relação de uma abordagem pedagógica e o aprendizado do aluno, descobrir fatores que influenciam o aprendizado, analisar comportamento do aluno evasor, dentre outras situações (Baker, Isotani e Carvalho, 2011; Costa et al., 2012; Webber, Zar e Lima, 2013; Rigo et al, 2014).

Para Romero, Ventura e García (2008) a MDE pode ser aplicada para melhorar o aprendizado, ao prover cursos adaptativos e com recomendação baseada no comportamento do aluno, além de poder fornecer feedback personalizado aos alunos e professores em várias situações.

Apesar de algumas ferramentas educacionais e AVEA oferecerem relatórios aos professores, tutores e gestores o volume de dados gerado é muito grande, tornando difícil extrair informações úteis somente através destes relatórios (Gaudioso e Talavera, 2006). Além disso, os relatórios que podem ser apresentados diretamente pelas ferramentas, apesar de possuírem informações relevantes, podem ser considerados superficiais, diante da quantidade de dados que estão armazenados e, muita das vezes pode tornar árduo para o professor extrair informação útil através da junção dos relatórios disponíveis (Romero, Ventura e Garcia, 2008). Sendo assim, através da aplicação de MDE obtêm-se dados mais significativos ao propósito desejado.

Porém, para a mineração se efetivar é preciso passar por várias etapas que compreendem o processo de descoberta de conhecimento como pode ser observado na figura 2.

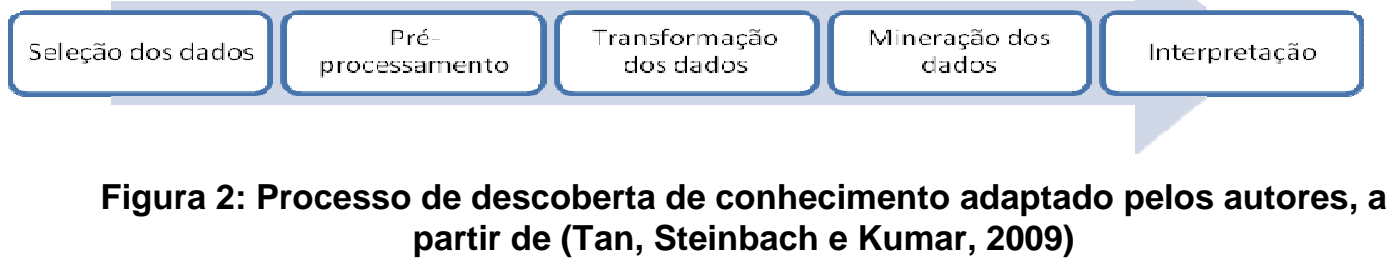

Para seguir as etapas supracitadas é preciso saber, inicialmente, qual é o objetivo que se pretende alcançar a fim de escolher técnicas e métodos mais adequados. Pela taxonomia proposta por Baker, Isotani e Carvalho (2011) os métodos são: predição, agrupamento, mineração das relações, destilação de dados para facilitar as decisões humanas e descoberta com modelos. Diante do objetivo da presente pesquisa, ao almejar fazer uma classificação de alunos desanimados, contempla-se o aprendizado de máquina supervisionado, ao obter um modelo para essa classificação e predição.

Cabe ressaltar que a predição é uma técnica usada para prever um valor futuro de um determinado atributo visando desenvolver modelos que deduzam aspectos 
específicos de dados. Utilizam variáveis da base de dados para prever valores desconhecidos ou valores futuros de outras variáveis de interesse, ou seja, através da predição que se consegue modelos para apoio à inferência a partir da associação dos dados existentes na base (Costa et al., 2012; Rigo et al, 2014). Costa et al, (2012) destacam que através da predição é possível verificar quais dados são mais importantes para o modelo.

As técnicas usadas na predição são classificação e regressão. Para usar o modelo classificador de predição, é preciso trabalhar com duas bases de dados, uma base de treinamento e uma base de teste. Tan, Steinbach e Kumar (2009) explicam que o modelo do conjunto de treinamento é usado para construir um modelo de classificação, que subsequentemente é aplicado ao conjunto de testes, sendo que a avaliação de seu desempenho é baseada na contagem de registros de testes previstos correta e incorretamente. Uma das técnicas de classificação simples e bastante usada é árvore de decisão.

Árvores de decisão é um exemplo de modelo estatístico que usa classificação e predição de dados, além de ser um dos algoritmos mais usados para predição. Dentre os algoritmos de árvore de decisão optou-se pelo uso do J48, visto ser um dos algoritmos que geram melhor resultado para árvores de decisão, além de se mostrar adequado para procedimentos envolvendo variáveis contínuas e discretas conforme mencionado por Librelotto e Mozzaquatro (2013).

\section{Trabalhos relacionados}

Pesquisas que envolvem MDE são aplicadas em diferentes contextos e finalidades (Baker, Isotani e Carvalho, 2011; Rigo et al., 2014). Particularmente, pesquisas que associam MDE e Computação Afetiva na busca por modelos e/ou aplicações, com finalidades em aprofundar estudos sobre fenômenos afetivos na educação estão em crescimento (Baker, Isotani e Carvalho, 2011; Romero et al., 2006).

A pesquisa desenvolvida por Iepsen (2013) utilizou a MDE para detectar padrões de comportamento associados com o estado afetivo de frustração no aluno. $\mathrm{O}$ autor analisou variáveis comportamentais do aluno dentro de um ambiente para ensino de algoritmos, a fim de identificar o aluno frustrado, buscando minimizar desistência na disciplina. A técnica aplicada foi classificação baseada em regras. Em nossa pesquisa, o foco está no aluno desanimado e vale ressaltar que, de acordo com Scherer(2005) o estado de ânimo desanimado engloba famílias e termos afetivos aos quais incluem a frustração. Além disso, a técnica escolhida foi a classificação a fim de prever o aluno propenso ao desânimo e tomar ações para recuperar esse aluno antecipadamente.

Majadas, Santos e Boticario (2013) também têm dedicado esforços para a predição do estado afetivo positivo ou negativo de alunos em ambientes educacionais. Os autores realizaram um experimento onde os alunos resolveram problemas de matemática e foram monitorados e coletados dados como tempo médio de pressionar e soltar teclas do teclado, detecção de suas expressões faciais com apoio do kinect e de um especialista da área, bem como usaram ao final de cada atividade um auto-relato usando questionário SAM(Self-Assesment Manequin), que posteriormente foi analisado 
juntamente com o apoio de especialista. De posse dos dados dessas diversas fontes, os dados foram minerados usando árvore de decisão e redes bayesianas. Uma-diferença com relação ao trabalho aqui descrito é que o questionário de auto-relato usado não exige um especialista da área de Psicologia, conforme apontado por Scherer(2005), o que propicia maior autonomia no processo de predição, bem como as variáveis analisadas são vindas do $\log$ do aluno no ambiente, o que a torna menos invasiva por não ter uso de sensores.

\section{Procedimento Metodológico}

A pesquisa em desenvolvimento é de natureza aplicada ao ensino e aprendizagem, com foco em disciplinas que utilizam AVEA o que permite o acesso aos dados das interações do aluno. É classificada como explicativa e, com relação aos procedimentos técnicos, foi desenvolvido um experimento onde participaram duas turmas de alunos da disciplina de Análise de Demonstrações Contábeis ofertada pelo Departamento de Ciências Contábeis e Atuárias da UFRGS.

Como o foco da pesquisa é predizer alunos propensos ao desânimo através do comportamento observável do aluno em interação em um AVEA foram aplicados dois questionários para obter-se variáveis relacionadas à percepção do aluno quanto a sua trajetória de aprendizado tanto da operação das funcionalidades e interação no ambiente.

Sendo assim, o primeiro questionário abordou as questões de como o aluno sentia em diversas situações de aprendizagem através da REA 2.0 (detalhes na seção 2.1). A REA 2.0 foi implementada utilizando linguagem PHP e banco de dados MySql e encontra-se disponível no link: http://pesquisacomputacaoafetiva.esy.es/moodle lindex.php. O segundo, complementar ao primeiro, contou com questões sobre dificuldade em usar ferramentas, formas de apoio que o professor poderia usar com a turma, formas de solicitação de ajuda ao professor, envio de atividades individuais e em grupo, acompanhamento da disciplina.

Após a aplicação dos questionários, os resultados foram tabulados, préprocessados e transformados para aplicar a mineração. Dessa forma, o início do processo de descoberta de conhecimento foi aplicado ao presente trabalho: a seleção dos dados se deu a partir do AVEA Moodle, onde foram selecionados os dados dos $\log s$ dos alunos e as questões dos questionários. Posteriormente, foi feito o pré-processamento desses dados, o que exigiu uma limpeza dos dados, deixando os dados somente dos alunos que se propuseram participar da pesquisa, eliminando redundâncias. Foram aplicados métodos estatísticos utilizando o software SPSS como histogramas de frequiência, foi feita a discretização dos dados, geração de quartis, tabulações cruzadas, dentre outras, a fim de encontrar as variáveis mais significativas e preparar os dados para a mineração. A próxima etapa, a transformação dos dados, dedicou-se a transpor os dados para o tipo de arquivo a ser utilizado pelo minerador WEKA (Waikato Environment for Knowledge Analysis), ou seja, os dados foram transformados em arquivos do tipo ARFF (Atribute Relation File Format), e adaptados para o algoritmo utilizado.

Somente após as etapas supracitadas é que as etapas de mineração e de interpretação foram realizadas e são descritas na seção 5 . 


\section{Análise e resultados do experimento}

Esse experimento contou com uma amostra de 44 alunos que participaram dos dois questionários e que puderam ter seus dados de $\log s$ analisados durante o semestre de 2014/2. Foram coletadas 26.611 interações nas mais diversas funcionalidades do Moodle para as turmas A e B.

Os componentes de log de maior participação foram: arquivos (incluem todos os documentos que o professor posta no ambiente ) com total de 3585 participações; fórum (incluem todos os fóruns criados) com um total de 2622 participações; sistema (contabiliza os acessos ao sistema) com 10140 acessos e, tarefa (incluem as visualizações, postagens de atividades, envio de arquivos das tarefas, confirmação de envio de tarefas) com um total de 5557 participações.

Sendo assim, a partir dessa análise optou-se por minerar dados dos fóruns e dos questionários aplicados devido às interações e ações realizadas. Ao analisar os $\log s$ dos alunos com relação ao fórum, constatou-se que os logs dos fóruns se dividiram em 3 segmentos (Fórum avisos da professora; Fórum Contatos com a Monitoria e Fórum dos grupos de trabalho), onde somaram respectivamente 404, 149 e 1973 acessos. O $\log$ de cada fórum é composto por: fórum_add_post, fórum_view_discussion, fórum_view_forum, fórum_view_discussion, fórum_add_discussion, dentre outros, o que gerou mais de 15 atributos somente relacionados aos fóruns.

Com relação aos questionários aplicados, o questionário que usou a REA 2.0 foi composto por 7 questões, porém foram consideradas para a mineração as três questões iniciais 1: $O$ que você sente quando envia atividades individuais em atraso; 2: $O$ que você sente quando envia atividades em grupo com atraso?; $O$ que você se sente quanto tem dificuldades em realizar atividades individuais? Para cada questão o aluno escolheu a família afetiva e a intensidade sentimento.

Com relação ao segundo questionário, as questões selecionadas para mineração abordavam a frequência com que o aluno enviava atividades individuais/grupo em atraso e se sentia dificuldades em realizá-las, além de questões relacionadas ao conteúdo, questionando se o aluno consegue acompanhar o conteúdo e se teve dificuldade com algum conteúdo na disciplina. Essas questões utilizaram a escala Likert (nunca, raramente, algumas vezes, quase sempre e sempre) como opção de resposta para as perguntas. A escolha das questões se deu pelas tabulações cruzadas encontradas entre elas através do software SPSS às quais mostraram-se relevantes para o estudo.

Após passar pelas etapas iniciais da descoberta de conhecimento, desde a seleção dos dados até a transformação desses dados, a mineração pode, então, ser realizada. $\mathrm{O}$ método usado na etapa de mineração foi classificação e foi feito utilizando aprendizado supervisionado através do algoritmo de árvore de decisão J48. Foi usado o método Holdout, apesar da quantidade limitada de dados de 44 registros, onde os dados foram separados em dois conjuntos, um conjunto de treinamento (21instâncias) e um conjunto de testes (23 instâncias).

Por fim, a etapa de interpretação foi feita pelo especialista da área e usada para suporte de tomadas de decisão na disciplina em questão. Sendo assim, com o conjunto 
de treinamento foi possível classificar corretamente $95,45 \%$ dos dados. O coeficiente Kappa obtido teve escore de $0,92 \%$. O coeficiente Kappa é usado como uma medida para descrever e testar a confiabilidade e precisão gerando o grau de concordância na classificação. Valores Kappa acima de 0,75 representam excelente concordância (Perroca e Gaidzinski, 2003).

O resultado obtido para o conjunto de teste, representado pela figura 3 , mostra que com esse conjunto foi possível classificar corretamente cerca de $91 \%$ dos casos. $\mathrm{O}$ coeficiente Kappa apresentou escore de 0,85. Juntamente a esses dados, o minerador gerou a matriz de confusão, que auxilia na avaliação do desempenho de um modelo de classificação e é baseada nas contagens de registros de testes previstos correta e incorretamente pelo modelo e, essas contagens são apresentadas através da matriz de confusão (Tan, Steinbach e Kumar, 2009).

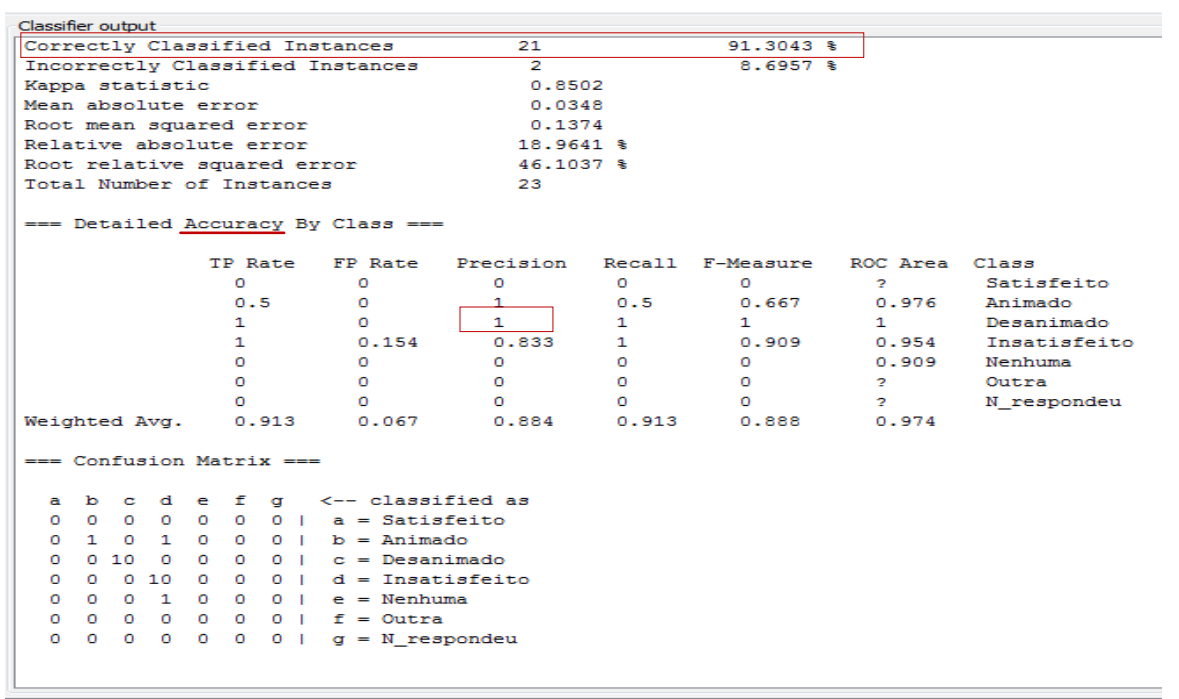

Figura 3: Resultado da mineração J48 - base de teste

A matriz de confusão apresentou classes "Satisfeito, Animado, Desanimado, Insatisfeito, Nenhuma, Outra e N_respondeu" onde as classes que apresentam o valor zero (0) em suas linhas não foram classificadas devido a esse modelo de testes não ter tido nenhuma resposta para esses valores. Uma opção seria descartar esses valores para obter uma melhor classificação, porém, o modelo de treinamento possuía respostas com as classes "Satisfeito, Outra e N_respondeu", o que iria gerar uma incompatibilidade com os dois conjuntos.

Sendo assim, das 23 instancias do conjunto de teste, 22 foram classificadas corretamente, que inclui as classes Desanimado, Insatisfeito e Nenhuma, às quais obtiveram valores verdadeiro/positivos. Porém, a classe Animado apresentou 01 instância falso/positivo, o que demonstra que um registro foi classificado incorretamente para a classe d(Insatisfeito). Quanto à acurácia do modelo, que pode ser observada em destaque na figura3, observa-se que a precisão apresentou resultados satisfatórios para o caso do aluno desanimado (100\%), que é o foco do presente estudo. Apesar da limitação da base de dados, seus resultados mostraram-se representativos nesta amostra, para a predição do desânimo, o que não descarta a necessidade do modelo ser reavaliado com um conjunto maior de dados. 


\section{Conclusão}

O presente trabalho teve como objetivo predizer o estado de desânimo de alunos em interação em um AVEA a partir da classificação dos seus dados e interações, fomentando a importância em considerar estados afetivos que podem levar à desistência do aluno no curso. A partir da aplicação da MDE, passando por todas as suas fases, foi possível classificar com uma taxa de acerto $91 \%$ dos alunos propensos ao desânimo.

Apesar do conjunto de dados do experimento ser considerado pequeno, os resultados mostraram-se relevantes, o que motiva novos experimentos em conjuntos de dados maiores a fim de averiguar o modelo, mantendo as mesmas variáveis.

Pretende-se em trabalhos futuros, subsidiar o professor com essas informações, apresentado de forma simples e clara os alunos propensos ao desânimo. De posse dessas informações, pode o professor adotar ações mais personalizadas que apóiem esse aluno na retomada de suas atividades e estudo e, propiciando a diminuição da desistência do curso, fato que é uma problemática na educação. Também pretende-se minerar os componentes Tarefas e Arquivos juntamente com os Fóruns, a fim de construir um novo modelo, com maior abrangência de dados comportamentais.

\section{Referencias}

Baker, R. S. J.; Isotani, S.; Carvalho, A. M. J. B. (2011). Mineração de Dados Educacionais: Oportunidades para o Brasil. Revista Brasileira de Informática na Educação. V.19, N.02.

Bercht, M. (2001). Em Direção a Agentes Pedagógicos com Dimensões Afetivas. Tese de doutorado. Tese de doutorado. Universidade Federal do Rio Grande do Sul. Programa de Pós-Graduação em Computação.

Costa, E.; Baker, R.; Amorin, L.; Magalhães, J.; Marinho , T. (2012). Mineração de Dados Educacionais: Conceitos, Técnicas, Ferramentas e Aplicações. Jornada de Atualização em Informática na Educação - JAIE.

Damando, F. S.; Bercht, M. (2014) Análise da disposição afetiva do aluno em um AVEA: descoberta do desânimo. XXV Simpósio Brasileiro de Informática na Educação.

Gaudioso. E.; Talavera, L. (2006). Data mining to support tutoring in virtual learning communities: experiences and challenges. In Data mining in e-learning. WIT Press.

Iepsen, E. F. (2013). Ensino de Algoritmos: Detecção do Estado Afetivo de Frustração para Apoio no Processo de Aprendizagem. Tese de Doutorado. Universidade Federal do Rio Grande do Sul. Programa de Pós-Graduação em Informática na Educação.

Inventado, P. S.; Legaspi P. Cabredo, R.; Numao, M. (2013) Modeling Affect in Student-driven Learning Scenarios. The $6^{\text {th }}$ International Conference on Educational Data Mining.

Jaques, P. A.; Nunes, M. A. S. N. (2012). Ambientes inteligentes de aprendizagem que inferem, expressam e possuem emoções e personalidade. Jornada de Atualização em Informática na Educação. $23^{\circ}$ Congresso Brasileiro de Informática na Educação. 
Librelotto, S. R.; Mozzaquatro, P. M. (2013). Análise dos Algoritmos de Mineração J48 e Apriori Aplicados na Detecção de Indicadores de Qualidade de Vida. Revint Revista Interdisciplinar de Ensino, Pesquisa e Extensão V.01, N.01.

Longhi, M. T. (2011). Mapeamento de Aspectos Afetivos em um Ambiente Virtual de Aprendizagem. Tese de doutorado. Universidade Federal do Rio Grande do Sul. Programa de Pós-Graduação em Informática na Educação.

Majadas, S. S.; Santos, O.; Boticario, J. G. (2013) Affective state detection in educational systems through mining multimodal data soucers. The $6^{\text {th }}$ International Conference on Educational Data Mining.

Pardos, Z, A.; Baker, R. S. J. D.; Pedro, M.S.; Gowda, Sujith. M.; Gowda, Supreeth, M. (2014). Affective States and State Tests: Investigating How Affect and Engagement during the School Year Predict End-of-Year Learning Outcomes. In Journal of Learning Analytics. V.01, N.01.

Perroca, M. G.; Gaidzinski, R.R. (2003). Avaliando a Confiança Interavaliadores de um Instrumento para Classificação de Pacientes - Coeficiente Kappa. Revista da Escola de Enfermagem USP. V. 37, N. 1. Scielo.

Picard, R. W. (2000). Affective Computing. The MIT Press.

Rigo, S. J.; Cambruzzi, W.; Barbosa, J. L. V.; Cazella, S. C. (2014). Aplicações de Mineração de Dados Educacionais e Learning Analytics com foco na evasão escolar: oportunidades e desafios. Revista Brasileira de Informática na Educação. V. 22, N.01.

Romero, C. M.; Ventura, S.; García, E. (2008). Data Mining in Course Management Systems: Moodle Case Study and Tutorial. Computers \& Education 51, pg. 368-384, Elsevier.

Sacharin, V.; Schlegel, K.; Scherer, K. R. (2013). Geneva Emotion Wheel Rating Study. Geneva, Switzerland: University of Geneva, Swiss Center for Affective Sciences.

Scherer, K. R. (2005). What are the emotions? And how can they measured? In: Social Science Information.

Tan, Pang-Ning; Steinbach, M.; Kumar, V. (2009). Introdução ao DataMining Mineração de Dados. Rio de Janeiro: Editora Ciência Moderna Ltda.

Webber, C. G.; Zat, D.; Lima, M. F. W. P. (2013). Utilização de algoritmos de agrupamento na mineração de dados educacionais. Revista Renote: Novas tecnologias na educação. V.11, $\mathrm{N}^{\mathrm{o}} 1$. 